\title{
SUPERCONDUCTING FINAL FOCUS FOR THE SLAC LINEAR COLLIDER*
}

DE92

\author{
W. W. Ash, F. Barrera, W. Burgess, K. Cook, H. Cutler, J. Ferrie, H. Petersen, D. Sawyer and R. Rinta
}

Research Division, Stanford Linear Accelerator Center, Stanford University, Stanford, California 94309

\begin{abstract}
Triplets composed of superconducting quadrupoles have been built and installed as the final focusing element for the high-energy positron and electron beams of the SLAC Linear Collider. Special features include independent alignment to 100micron tolerance inside a common cryostat; non-magnetic materials to allow operation inside the detector's solenoid field; a continuous-flow helium-only system using 50-meter-long flexible transfer lines; and complete operation of the system before installation. The mechanical design and cryogenic operation experience are presented.
\end{abstract}

\section{INTRODUCT,ON}

The Superconducting Final Focus was designed to solve several novel and difficult requirements posed by the experimental program at the SLAC Linear Collider. Machine optics require high-gradient $(120 \mathrm{~T} / \mathrm{m})$ precisely aligned magnetic quadrupoles to focus the $50 \mathrm{GeV} / \mathrm{c}$ electron and positron beams to few-microndiameter spots[1]. The new physics experiment for this facility, the SLC Large Detector (SLD), requires focusing elements that can work in the presence of its 6-Tesla solenoidal field and with as small an encumbrance of the detector area as possible. Finally, this new focusing system had to be made ultra reliable with all controls remoted, be completely tested and aligned in final form offline, and then installed with as few changes as possible.

These criteria guided the design of the focusing system as follows:

The high-gradient and external-field conditions were met by using superconducting quadrupoles with nonmagnetic materials. Precision alignment was achieved by pinning the magnet coils and laminations into stiff cylinders which formed part of the cryostat. These cylinders were adjusted from the outside through lowloss gimbals and supports while monitoring the field position through a probe in a warm bore on the magnet axis.

The reliability, testing, and installation issues were solved by using simple pool-boiling helium-only cryustats continuously fed by flexible, low-loss transfer lines.

A schematic overview of the system is shown in Figure 1. It has been successfully operating at the SLC since early 1991.

\section{MAGNETS/CRYOSTATS}

Each of the three quadrupole magnets, which were designed and fabricated at Fermi National Laboratory [2], is precisely located, pinned and welded inside, close-toleranced, mechanically stiff, machined 304L stainless steel tubes $(600 / 1200-\mathrm{mm}$ long, $170-\mathrm{mm}$ OD $\times 7.6-\mathrm{mm}$ wall), which also form part of the helium vessel. The three vessels/magnets are interconnected by $180-\mathrm{mm}$ OD stainless steel bellows which allow independent movement. The $44-\mathrm{mm} \mathrm{OD} \times 1.5-\mathrm{mm}$ cold bore is constructed similarly.

Each helium vessel is fixed at its center by an epoxy composite gimbals, which locates and retains each vessel/magnet along the magnetic axis while allowing sufficient transverse freedom, (Fig. 2a).

"Work supported by Department of Energy contract DE-AC03-7676SF00515.

Presented at the 14th International Cryogenic Engineering Conference, Kiev, Ukraine, June 8 - 12,1992 
Precision transverse alignment of each individual magnet inside the $3800-\mathrm{mm}$ long, $330-\mathrm{mm}$ OD $\times 25.4$ $\mathrm{mm}$ wall, stainless steel vacuum vessel is made by four ( 2 horizontal, 2 vertical), low heat load, positive displacement, screw action adjusters, each acting against an opposing return spring, (Fig. 2b,c). These pusher/adjusters accommodate all axial and transverse thermal contractions and a vertical sag of 6 - $\mathrm{mm}$ from the cantilevered cryostat each time the detector end cap door, which forms part of the front external support, is opened. A schematic of the positional tolerances for the triplet magnets is shown in Fig. 3. The three magnets were aligned to within $>50 \mu \mathrm{m}$ with the system cold and powered, with little or no displacement following thermal cycles or mechanical movement.

\section{CRYOGENICS}

Due to space restrictions in the experimental hall pit, the CTI-4000 liquefier, supply dewar and distribution valve box are located external to these areas at ground level. A gas/liquid phase separator is incorporated in the valve box to minimize the helium vapor fraction introduced by the dewar supply line, valves and valve box. This vapor is used to cool the valye box screen.

Liquid helium is supplied via low loss, flexible, coaxial transfer lines, of the type developed by CERN/Kabelmetal[3,4]. These lines are 58 and $76-\mathrm{m}$ long, each having a vertical drop of $18-\mathrm{m}$. The quality of the liquid helium delivered to the cryostat disconnect box is such that no further phase separation is required. The helium vessel and disconnect box are thermally shielded by vapor cooled, copper radiation screens. A schematic of the system is shown in Fig 4.

Condensation on the $5000 \mathrm{~A}$ vapor cooled current leads is eliminated by mounting 2 temperature controlled, $200 \mathrm{~W}$, cartridge heaters inside each lead connector block, an important consideration since access for ice/water removal or maintenance would be highly disruptive.

Cooldown of the triplets to $100 \mathrm{~K}$ is carried out by passing liquefier supply gas, at a pressure of 2 bar, via a heat exchanger located in the liquefier $\mathrm{LN}_{2}$ supply dewar through the valve box and flexible lines to the triplet disconnect box/liquid reservoir. The gas passes from the disconnect box, through the cryostat helium vessel and magnets to exit at the vessel end, returning via the cryostat heat shields and external heat exchangers to compressor suction. During cooldown only a small fraction of gas $\left(501 \mathrm{~min}^{-1}\right)$ is returned via the transfer line shield. Progressively closing a manually operated bypass valve across the $\mathrm{LN}_{2} / \mathrm{He}$ heat exchanger permits uncomplicated and effective control of cooldown temperature and rate. Warm up is the reverse of the above procedure. The process takes $<36$ hours.

Final cooldown and liquid helium fill from $100 \mathrm{~K}$, takes $<6$ hours. The disconnect box/reservoir levels are held at \pm 1.5 -mm via proportional control valves, located in the valve box, which maintain quasi constant helium fiow to each triplet. Cryogenic performance and operating parameters for all three triplets were practically identical and are summarized in Table 1.

The extraneous helium vapor resulting from the heat load, created by the gimbals, 24 pusher/positioners and small $O D$, is utilized effectively in the cryostat heat shield which has an outlet temperature of $75 \mathrm{~K}$.

\begin{tabular}{|c|c|c|c|c|c|}
\hline & \multicolumn{4}{|c|}{ CRYOSTAT HELIUM FLOW DISTRIBUTION $\left(1 \mathrm{~min}^{-1}\right)$} & TOTAL. \\
\hline $\begin{array}{l}\text { MAGNET } \\
\text { STATUS }\end{array}$ & $\begin{array}{l}\text { CURRENT } \\
\text { LEADS (2) }\end{array}$ & $\begin{array}{l}\text { CRYOSTAT } \\
\text { SHIELD }\end{array}$ & $\begin{array}{l}\text { TRANSFER } \\
\text { LINE SHIELD }\end{array}$ & $\begin{array}{l}\text { TOTAL } \\
\text { FLOW }\end{array}$ & $\begin{array}{c}\text { LIQ. He } \\
\left(\mathrm{l} \mathrm{h}^{-1}\right)\end{array}$ \\
\hline UNPOWERED & 160 & 50 & 160 & 370 & 29 \\
\hline (I) NOMINAL & 220 & 60 & 180 & 460 & 36 \\
\hline
\end{tabular}

Table 1. Cryogenic Operating Parameters 


\section{PERFORMANCE}

Three triplets, including one complete spare, were fully tested for almost a year, with their flexible transfer lines, and aligned on the pit floor adjacent to the beam line prior to installation.

All design criteria have been successfully satisfied. The triplets have been in almost continual operation since February 1991, with less than 10 hours beam downtime attributed to system malfunctions.

\section{ACKNOWLEDGMENTS}

We wish to express our gratitude to EFD Cryogenics Group, the SLAC engineering and technical staff and the SLD group members whose efforts made this work possible; critical contributions have been made by B. Abley, R. Belcinski, J. Cobb, D. Jensen, J. Jensen, D. Jones, H. Maxon, M. Nordby, P. Roybal, R. Taylor, J. White, and N. Whynott. Special thanks go to W. Kaminskas who supervised the construction and installatior. team.

\section{REFERENCES}

[1] Toge, N., et al., 'New final focus system for the SLAC linear collider,' SLAC-PUB-5552, May 1991.

[2] McInturff, A. D., et al ., 'The magnetic properties of the SLC intersection region superconducting quadropole triplets,' SLAC-PUB-4478, November 1987.

[3] Blessing, H., et al., 'High performance flexible cryogenic helium transfer lines,' Adv. Cryo. Eng., vol. $27,(1982)$, pp. 761-768.

[4] Blessing, $\mathrm{H}$., et al., 'Controlled downwards transfer of saturated liquid helium across large differences in elevation,' Proc. ICEC12, Butterworth 1988, pp. 222-226.

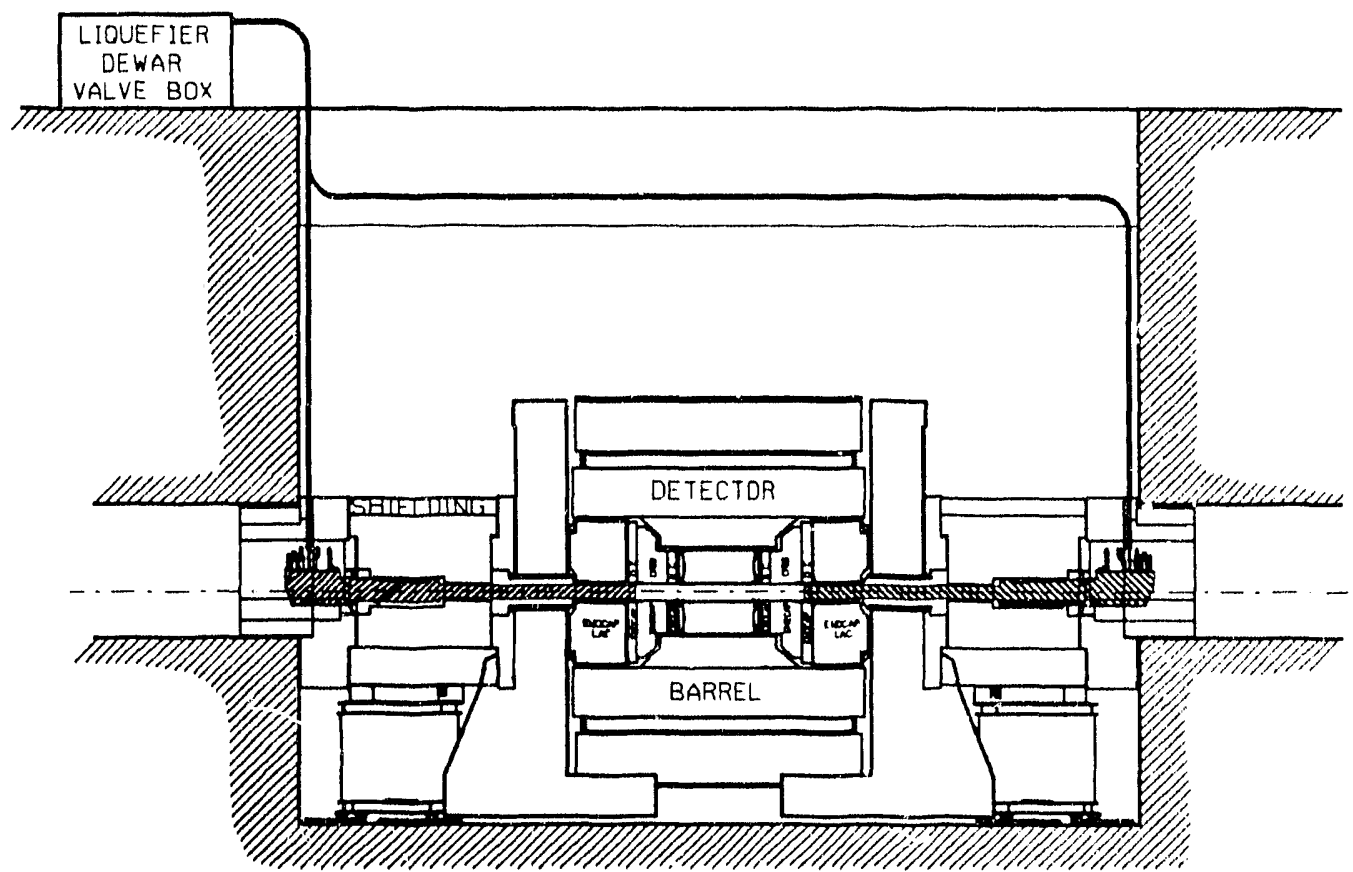

Figure 1. Triplet-detector layout. 

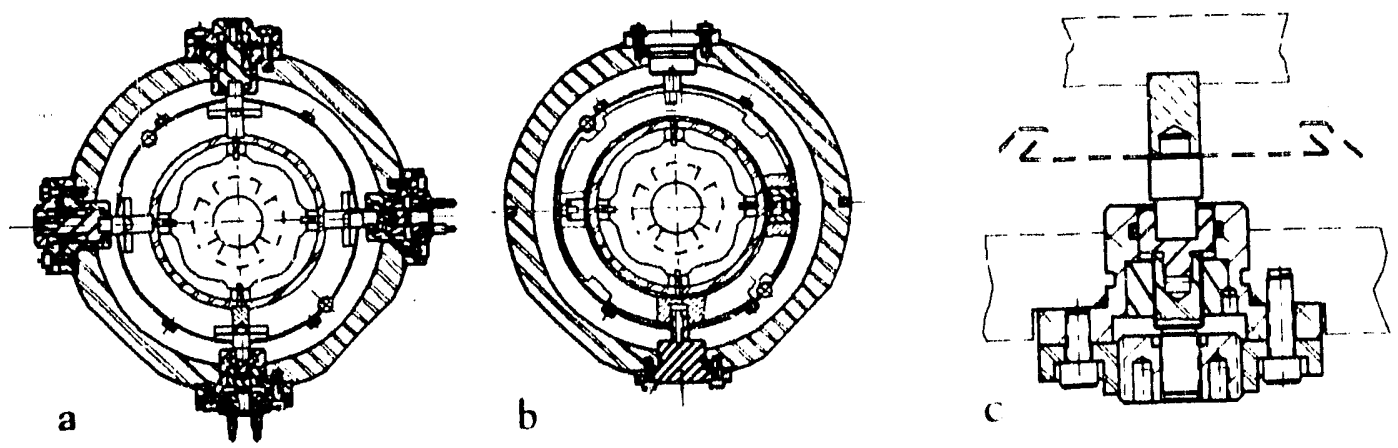

Figure 2. a) Adjuster-pusher; b) Gimbals; c) Screw adjuster.

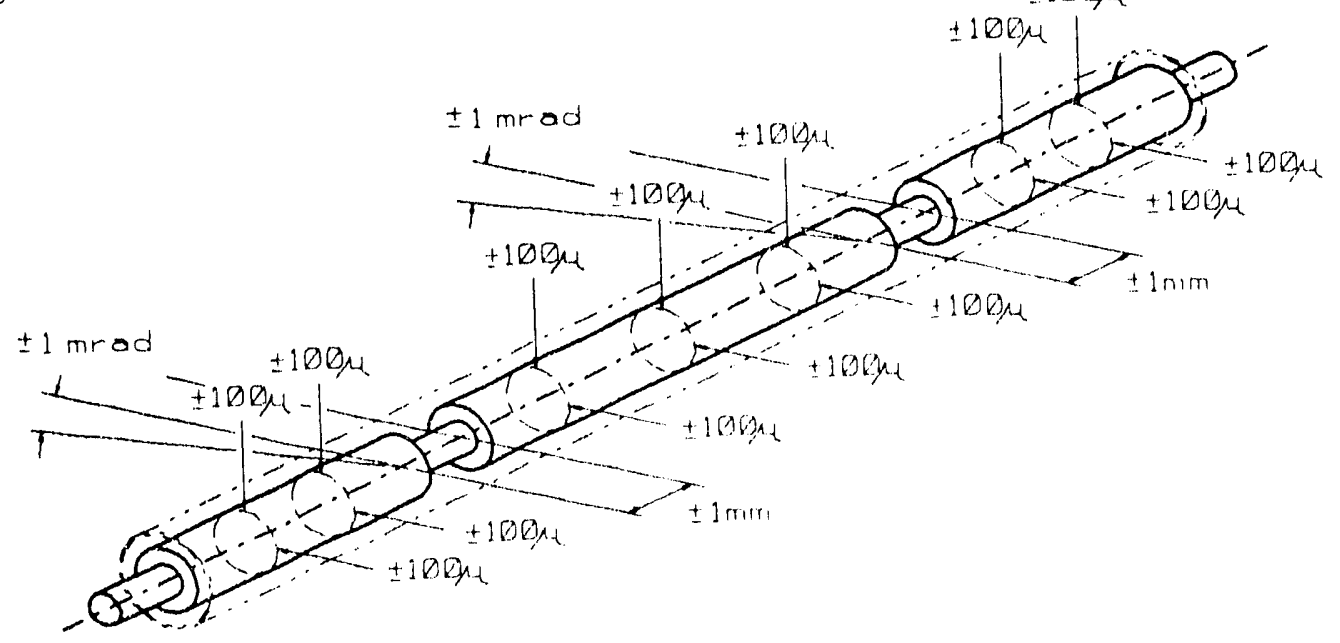

Figure 3. Static tolerances.

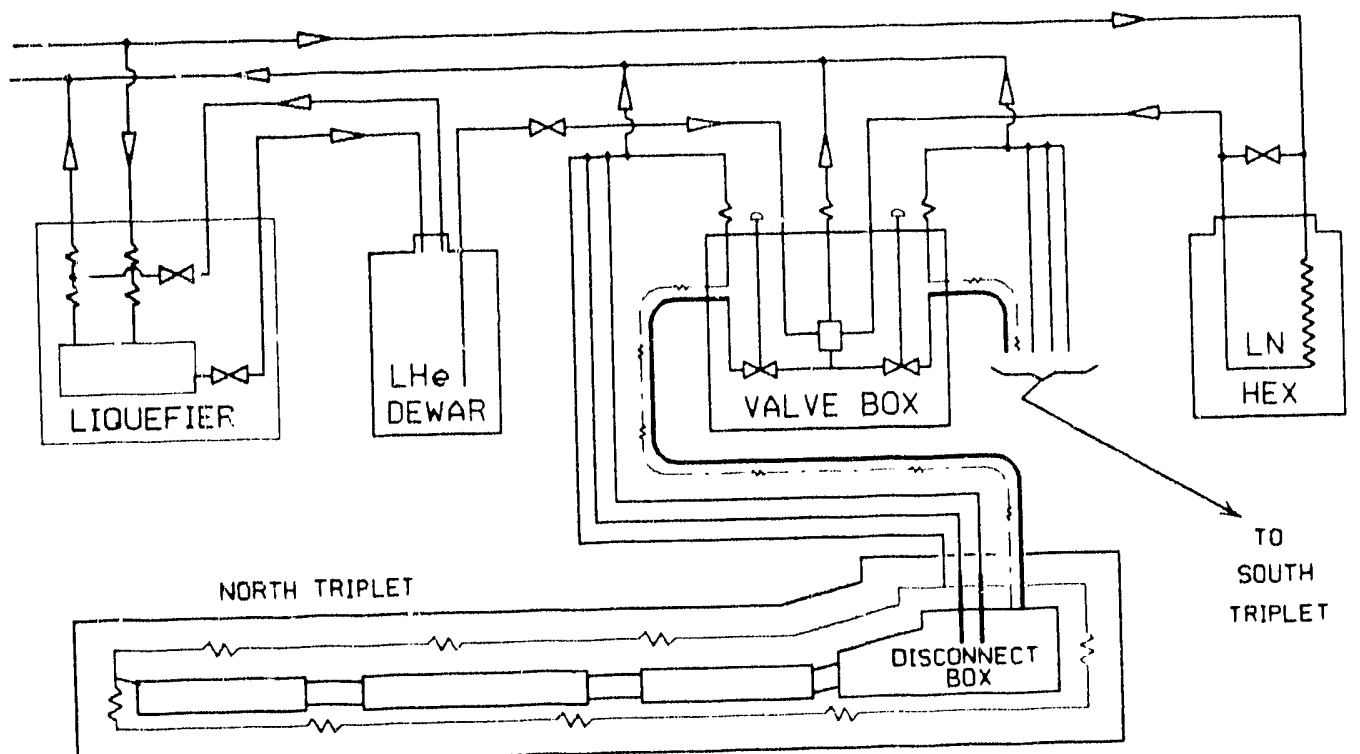

Figure 4. Cryogenis flow scheme. 


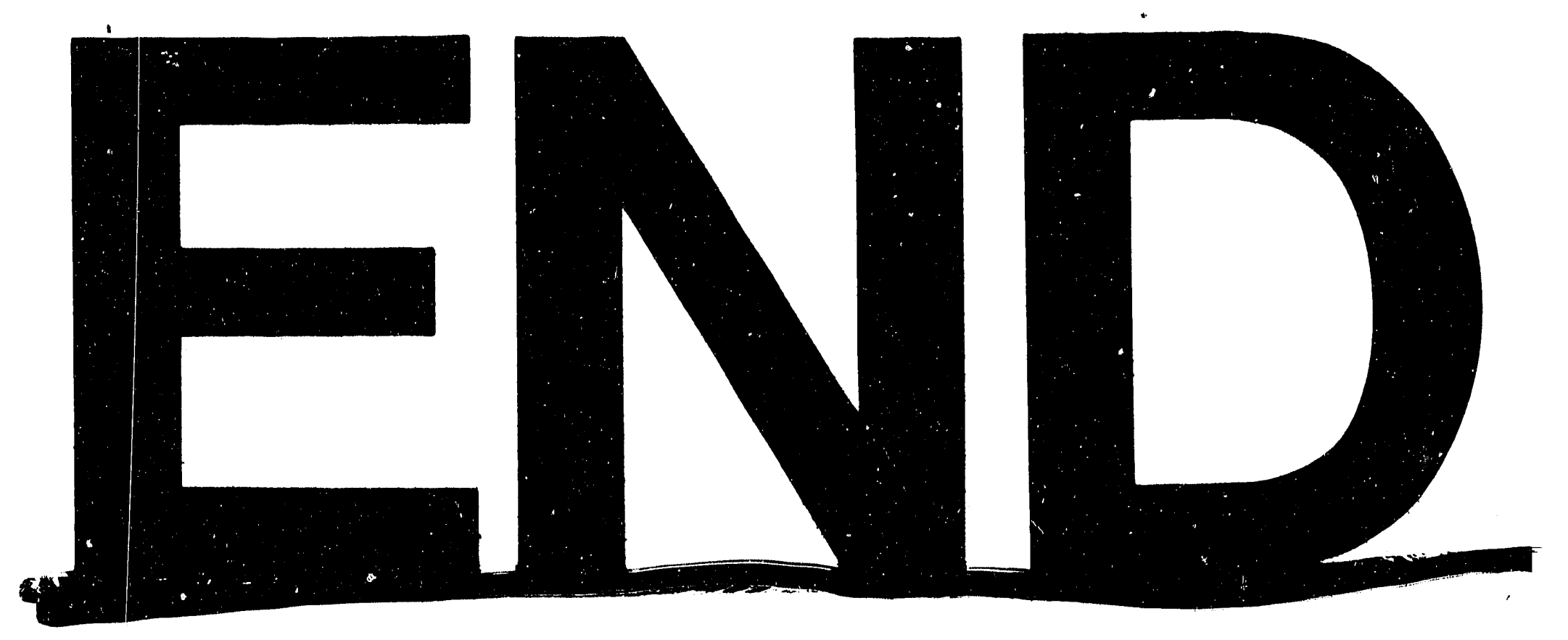

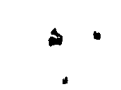
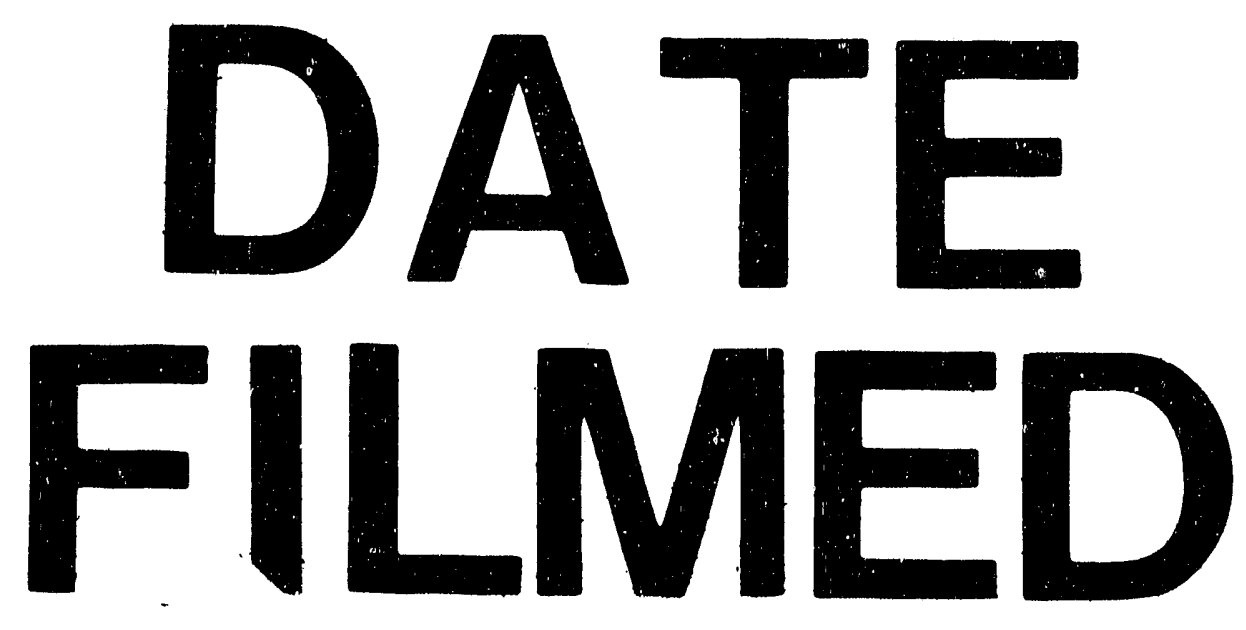

产

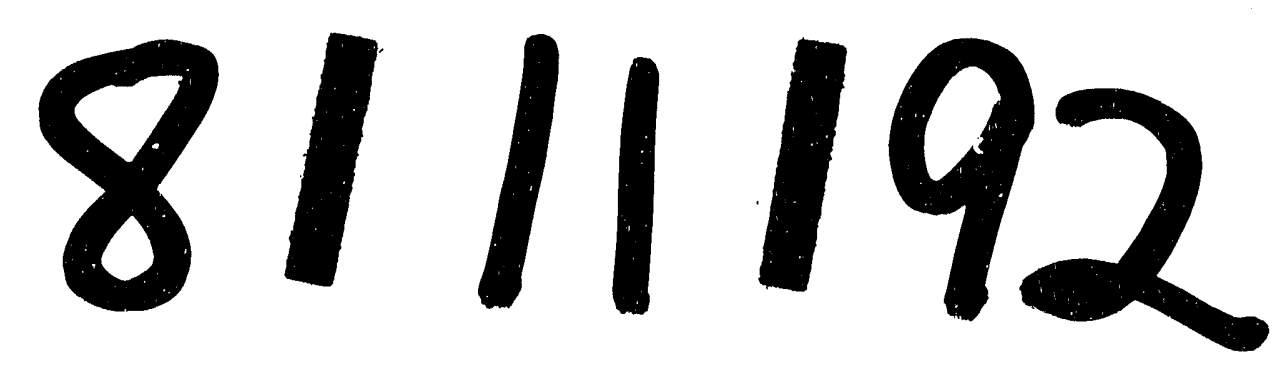


